\title{
EL CURRÍCULUM NULO Y SUS DIFERENTES MODALIDADES
}

\section{Beatriz M. Arrieta de Meza \\ Rafael Daniel Meza Cepeda \\ Universidad de Zulia, VENEZUELA}

\section{INTRODUCCIÓN}

En su obra "Análisis del Currículum" Posner (1998) nos presenta una tabla contentiva de lo que el autor denomina los cinco currícula simultáneos, ellos son:

1. Currículum Oficial: Descrito en forma documental, a través de planes y programas, materiales didácticos sugeridos, guías curriculares y los objetivos que el sistema educativo vigente aspire alcanzar mediante la aplicación de esos planes. La experiencia nos ha demostrado que el currículum oficial no es inflexible, ya que en la puesta en práctica de lo planificado intervienen diversos elementos humanos, materiales y circunstanciales que lo hacen dinámico, y por lo tanto, sujeto a modificaciones valederas.

2. Currículum Operacional: Currículum incorporado en las prácticas y pruebas de enseñanza reales; también denominado currículum pertinente (Arrieta y Meza: 2000), concebido como el resultado de la aplicabilidad y utilidad del currículum, cuando se pasa de la teoría (como estudiantes universitarios) a la práctica (en el desenvolvimiento como profesionales).

3. Currículum Oculto: Representado por las normas institucionales y valores no reconocidos abiertamente por profesores y funcionarios escolares; su profundidad e impacto a veces llegan a resultar mayores que los del currículum oficial.

4. Currículum Nulo: Tema de estudio no enseñado (Eisner, 1994), o que siendo parte del currículum no tienen aplicabilidad ni utilidad aparente, llegando a considerarse como materias y contenidos superfluos.

5. Extra Currículum: Son las experiencias planeadas, externas al currículum oficial, es de carácter voluntario y está vinculado con los intereses estudiantiles.

Al analizar estos tipos de currículum, encontramos que cuatro de ellos pueden ser verdaderos aportes al conocimiento, contribuyendo directamente al mejoramiento del proceso enseñanza-aprendizaje (o inter aprendizaje). Por el contrario, el llamado currículo nulo va a resultar ser una fuente de confusión y pérdidas de diversa índole, especialmente en lo referente a recursos humanos, materiales y financieros; su presencia constituye un factor definitivamente contaminante, desfavorable y dañino para los otros currícula.

En la investigación emprendida acerca de la pertinencia que tienen los estudios de lingüística en desempeño profesional, nos encontramos con casos de currículum nulo, evidenciados por las referencias que acerca de éste revelaron los sujetos de la investigación. Hemos decidido ampliar lo correspondiente al currículum nulo por considerarlo como uno de los factores que propician, en mayor grado, la generación de 
los problemas que aquejan actualmente a las instituciones educativas, especialmente en lo que respecta al resultado integral y último de la formación, es decir, cuando se llega al momento del ejercicio profesional.

Con el propósito de analizar el problema con mayor claridad, nos hemos planteado una serie de categorías para describir el currículum nulo, concebido por los autores de este artículo en una forma más amplia que la noción presentada por Eisner (1994) y Posner (1998).

\section{EL CURRÍCULUM NULO Y SUS VARIANTES}

Para abordar el tema del currículum nulo conviene traer a colación los cinco elementos conceptuales que deben tomarse en consideración para la elaboración del currículum (López, 1996) y su subsecuente puesta en práctica. Estos elementos son:

- Proceso de elaboración permanente y colectiva: Este proceso debe concebirse como una actividad dinámica, que sea el producto de la reflexión académica de profesores universitarios y egresados en ejercicio profesional de la investigación, donde la estructura curricular sea un medio científico más no un fin.

- Procesos vinculantes de la labor curricular: Estos procesos deben guardar una estrecha relación con la pertinencia y la operacionalidad del currículum, como el resultado de prácticas comprobadamente efectivas y satisfactorias.

- Pertinencia social y pertinencia académica: Tomando en consideración que el currículum debe tender a la búsqueda de respuestas a las necesidades reales de formación académicoprofesional del entorno social, el currículum debe concebirse con el propósito de que se logre la aplicabilidad real, efectiva y útil de lo planificado con una base sólida en el entorno. Al respecto conviene traer a colación a Carazza (1998) cuando recomienda que los académicos deben asumir la tarea política de participar en el proceso social, lo deseable es que esta participación sea colectiva, independientemente de que sea vulnerable, limitada, parcial, algunas veces acertada $u$ otras veces incorrectas, como todo lo humano.

- Participación, flexibilidad y practicidad: El currículum debe ser el resultado de una discusión participativa, sujeto a las modificaciones propias del paso de la teoría (currículum planificado) a la práctica efectiva (currículum operacional), coincidiendo con Marín (1995) vemos que en dicha práctica se observa que el currículum va más allá de las experiencias adquiridas por los estudiantes y de los objetivos propuestos por el sistema educativo.

- Interdisciplinariedad: Dada la complejidad del proceso de planificación curricular, se hace necesaria la participación de profesionales de diversas disciplinas del conocimiento, como educadores en las diferentes áreas, psicólogos, sociólogos, economistas, abogados y una representación de los verdaderos sujetos del currículum: los estudiantes universitarios, como futuros profesionales. 
A manera de síntesis, en el gráfico siguiente ofrecemos estos cinco elementos conceptuales del currículum, conducentes al logro del currículum pertinente.

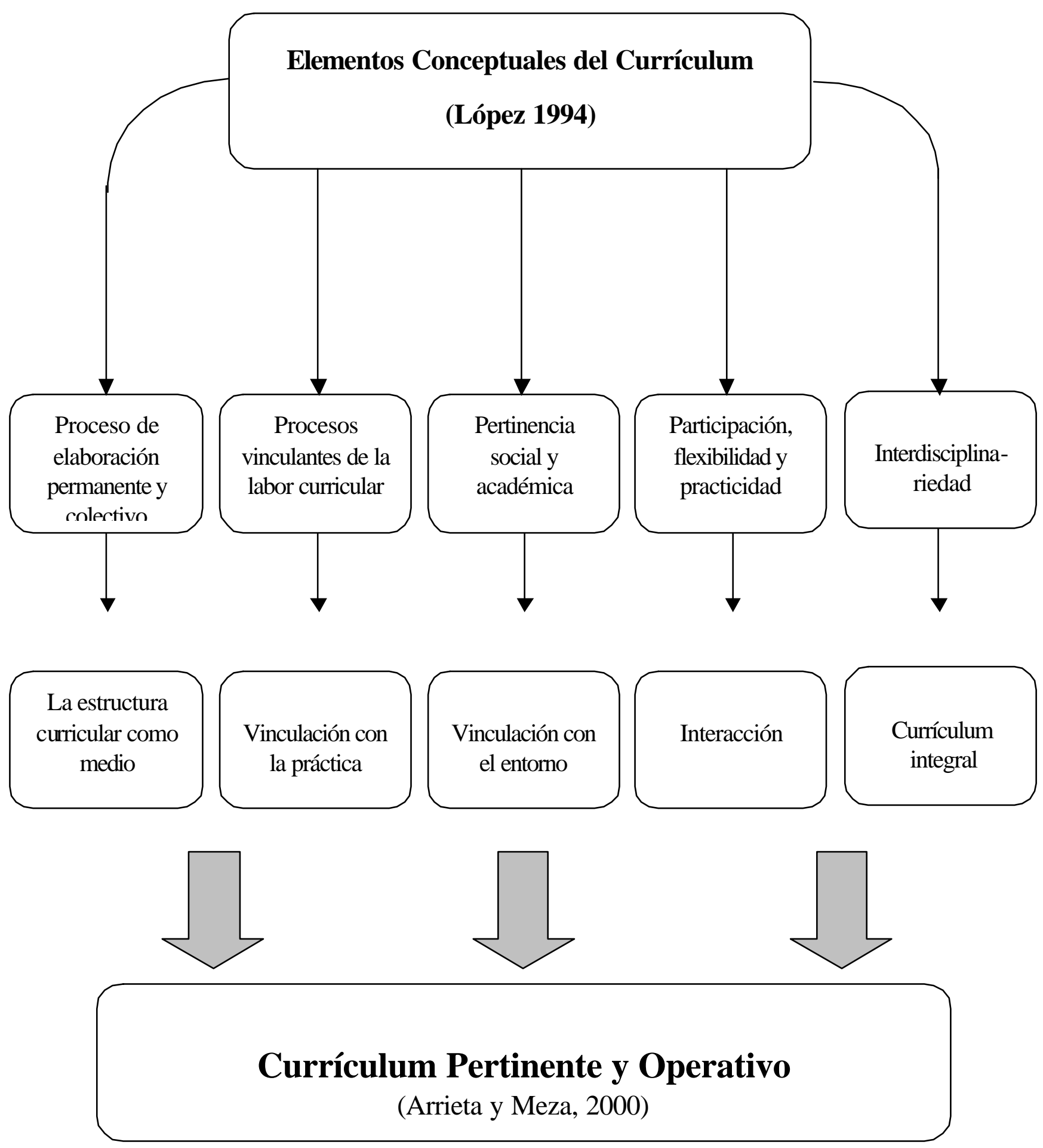


Como hemos podido observar, la interdisciplinariedad está estrechamente ligada a la consecución del currículum integral, por lo que coincidimos con Peñaloza (1996) cuando califica al currículum como el medio para concretar la noción de lo que es la educación. La no observación de la interdisciplinariedad en la planificación y ejecución curricular nos conduce a varias formas de currículum nulo, como veremos más adelante.

A través del desarrollo de la investigación relacionada con la pertinencia y operacionalidad del currículum, se ha podido determinar que al no tomar en cuenta los puntos conceptuales para la elaboración y subsecuente desarrollo del currículum, entre otros factores, se incurre en el llamado currículum nulo, del cual ofrecemos las siguientes categorías:

\section{CURRÍCULUM NULO POR OMISIÓN}

Se produce cuando no se incluyen en la programación importantes puntos necesarios para su aplicación durante el ejercicio profesional. También se da cuando en la estructura curricular de una carrera faltan una o varias asignaturas que agrupen dichos puntos fundamentales. Esta es la posición de Posner (1998), cuando hace mención a los temas de estudio no enseñados. Otro caso de currículum nulo por omisión se produce cuando, por diversas razones, aspectos que estaban contemplados en la planificación no se cumplieron, o fueron desarrollados en forma incompleta.

Son aquellos elementos de la programación, que no tienen aplicabilidad en el ejercicio profesional, esta inaplicabilidad puede darse por diversas razones, entre las cuales están la desvinculación de materias y contenido con el entorno profesional y social, y la obsolescencia de contenidos y fuentes manejadas y recomendadas por los docentes a los futuros profesionales.

\section{CURRÍCULUM NULO POR FRONDOSIDAD}

Se trata de elementos programáticos, demasiado ampliados en su diseño, los cuales no son necesarios dentro de lo fundamental del programa, en cuanto a su aplicabilidad durante el ejercicio profesional. Este tipo de currículum nulo se produce cuando se vulneran los principios de participación, flexibilidad y practicidad.

\section{CURRÍCULUM NULO POR REDUCCIÓN CRONOLÓGICA}

Son aquellos elementos que debieron ser elaborados durante un tiempo mayor, a cuyo aprendizaje deberían señalárseles un mayor número de horas. Generalmente los encontramos en prácticas de laboratorio, o en los diseños de asignaturas eminentemente prácticas.

\section{CURRÍCULUM NULO POR PREFERENCIA DEL DOCENTE}

OEI - Revista Iberoamericana de Educación 
Se refiere a aspectos que son detallados al máximo, con un gran consumo de tiempo por parte del docente, del estudiante y de la institución educativa en sí, por una preferencia manifiesta del docente a un tema específico, cuya aplicabilidad no se corresponde con la dedicación que se solicita del mismo.

\section{CURRÍCULUM NULO POR EVALUACIÓN INADECUADA}

Se refiere a la frustrante evaluación que resulta, cuando los elemento del conocimiento que integran el cuestionario de a misma, no fueron adecuadamente presentados durante el desarrollo del programa. El caso extremo está dado por una evaluación basada en elementos programáticos que no se consideraron durante el tiempo de desarrollo de la asignatura.

\section{CURRÍCULUM NULO POR FALTA DE INCENTIVO}

Se da cuando puntos importantes del programa diseñado, se ofrecen al discente con displicencia, falta de interés y hasta restándole importancia al contenido, cuando por el contrario, el contenido en cuestión, puede que sea imprescindible en el futuro ejercicio profesional.

\section{CURRÍCULUM NULO POR FALTA DE PREPARACIÓN DEL DOCENTE}

Se presenta cuando se encarga a un docente, una programación que desconoce, o para la cual está poco preparado, no siendo la mayoría de las veces, parte de su propia formación. El resultado para el estudiante de este tipo de exposiciones puede ser desastroso.

\section{CURRÍCULUM NULO POR DESFASE DEL CONOCIMIENTO}

Se produce cuando nos encontramos con conocimientos, aparentemente necesarios y de gran aplicabilidad, que son desarrollados sobre la base de exposiciones con contenido no actualizado u obsoleto.

\section{CURRÍCULUM NULO POR FALLAS METODOLÓGICAS}

El proceso enseñanza-aprendizaje (o Inter-aprendizaje), puede resultar afectado por el escaso conocimiento que tenga de la metodología el profesor encargado de una signatura. Este problema se presenta sobre todo en algunos especialistas de las ciencias, quienes no quieren tomar en cuenta las bases pedagógicas de la educación.

\section{CURRÍCULUM NULO POR EXCESO DE AYUDAS AUDIOVISUALES}

Es difícil pensar en algo más propicio a la distracción y falta de atención, que el recargar las exposiciones magistrales con material audiovisual de todo tipo, el cual algunos docentes utilizan, más con el fin de demostrar su experticia, que como el resultado de una adecuada planificación de una sesión de información o formación. 
CURRÍCULUM NULO POR DESFASE ENTRE LA PREPARACIÓN PREVIA DEL ALUMNO Y EL NUEVO MATERIAL

Especialmente en asignaturas con imprescindible prelación, encontramos alumnos a los cuales les faltan bases fundamentales, para la aprehensión del nuevo conocimiento. Cuando esto sucede, generalmente se recurre a los denominados cursos propedéuticos, pero por experiencia se sabe que esto no es factible en todos los programas universitarios. Las deficiencias por desfase se detectan generalmente en las pruebas diagnósticas que algunos profesores aplican antes de dar inicio a sus cursos, con el fin de planificar de acuerdo al grado de profundidad del conocimiento que tengan sus alumnos y saber en cuales aspectos hacer más énfasis.

\section{CURRÍCULUM NULO POR SUPERFICIALIDAD}

Se trata de programas o puntos de la programación, que son tratados en una forma muy superficial, cuando en el ejercicio profesional serían de gran interés por su aplicabilidad. La superficialidad puede obedecer a diversos factores; guarda relación con otros tipos de currículum nulo, como la falta de preparación adecuada del docente, apatía y desactualización, entre otros.

\section{CURRÍCULUM NULO POR FALTA DE EJERCICIO PROFESIONAL DE LA CARRERA ORIGINAL}

Las universidades sobre todo, han tenido la tendencia a formar y contratar personal docente que nunca se ha desempeñado como profesional de su carrera original. Por muy bien preparado que encontremos al docente, no puede enseñar con profundidad y conocimiento adecuado lo que nunca ha ejercido ante la comunidad social. Defendemos firmemente la figura del Becario Académico como manera de iniciar a los mejores egresados en la carrera docente universitaria, pero no la concebimos como una sucesión continua y eectiva de formación académica, si no lo enfrentamos a la realidad social de su profesión original, por lo menos durante dos años de ejercicio profesional. Después de los estudios de maestría, recomendaríamos un año de ejercicio profesional y otro adicional luego de los estudios del doctorado. Consideramos que ésta sería una manera real y efectiva de vincular prácticamente lo que se aprendió y lo que se necesita para el correcto ejercicio profesional. Nuestra experiencia nos indica que sería conveniente estudiar la posibilidad de que algunos años sabáticos, sean dedicados a un reencuentro social con la profesión original.

\section{CURRÍCULUM NULO POR NOVEDAD}

La inclusión de temas o hasta asignaturas completas, simplemente porque son novedosas, sin un previo estudio de su verdadera aplicabilidad, lleva a la confusión y hasta una total pérdida de tiempo y esfuerzo por parte del docente y del estudiante. Sobran ejemplos de tendencias o prácticas que aparecen como la gran panacea educativa, pero una vez llevadas al campo de la aplicación, no resultan más que una esperanza que no ayuda en nada. 


\section{CURRÍCULUM NULO POR TRADICIÓN}

Algunas asignaturas se mantienen en la programación de una carrera, únicamente porque se supone que siempre han estado o por un malentendido respeto al docente que las administra. No importa lo tradicional que pueda ser una asignatura, partes de ella o los temas que contiene, sino que tiene aplicabilidad por la forma en que es administrada, ésta representa una pérdida notoria en el proceso enseñanza aprendizaje.

\section{CURRÍCULUM NULO POR FALTA DE COORDINACIÓN}

Nos referimos en este caso a la repetición de temas, en diferentes asignaturas, por el simple hecho de que no hay coordinación entre los profesores de esas asignaturas, las cuales contienen elementos o temas comunes. Es bueno recordar que la falta de coordinación vertical (secuencias o prelaciones) y horizontal (asignaturas del mismo año o semestre), puede echar a perder un eje temático necesario para la formación y posterior uso de temas en el ejercicio profesional.

\section{CONSIDERACIONES FINALES}

Cualquiera que sea el tipo de currículum nulo que se esté desarrollando en una programación, esto trae pérdidas que podemos llevar al terreno de lo humano, lo físico y lo financiero, consecuentemente debemos proceder racionalmente a analizar este tipo de currículum nulo para su eliminación o por lo menos su control. Si analizamos un semestre regular de 16 semanas, con cuatro horas semanales, y analizamos el peor de los casos, asignaturas innecesarias, esto conlleva a una pérdida por parte del docente equivalente a 64 horas de asignatura, y si el dictado se hace a unos 30 alumnos, el número de horas perdidas por los alumnos llega a 1940 horas, y un total entre profesor y alumnos de 2004 horas íntegramente innecesarias. Considerando solamente una hora para preparación de clases o estudio de las mismas, la relación de la cual estamos hablando señala unas 4008 horas totalmente superfluas, en un semestre de una sola asignatura.

La pérdida por un tema innecesario de cuatro horas, puede resultar en el equivalente a 124 horas perdidas entre docente y discente, como mínimo. El mismo tipo de análisis antes comentado nos presenta 248 horas usadas sin ningún beneficio.

Se nos complica mucho más el caso, si tomamos en cuenta que el ac to docente se ha desarrollado en un entorno físico, dentro de un aula, laboratorio o taller, lo cual representa gastos de mantenimiento, subsistencia y reemplazo de insumos en la mayoría de los casos. Si queremos acercarnos a la realidad de la pérdida financiera, sencillamente calculemos el costo del profesor por hora multiplicada por el número de horas de la cátedra. A lo anterior se le sumará el costo de alumno por hora, multiplicado por el número de horas, y por el número de alumnos. Sumando también el valor económico que representa para la institución el costo del aula, según el número de horas en que ésta sea utilizada y todavía falta sumar costo de insumos didácticos, material, bibliografía utilizada, etc., lo que nos puede llevar a una cifra, verdaderamente 
preocupante.

Si volvemos un poco atrás, encontraremos que el currículum nulo, en forma parcial la mayoría de las veces, y total o casi total, en otras, debe su aparición, más que todo a elementos negativos, como lo pueden ser la ignorancia, la arrogancia o la insensatez. Corrijamos estos factores y fácilmente podemos analizar nosotros mismos lo nulo de nuestras propias programaciones; si vemos necesaria una corrección o varias, hagámosla de inmediato, y ofreceremos una programación que recordarán y aplicarán nuestros alumnos cuando ejerzan la profesión, porque lo que hemos enseñado tiene pertinencia por su aplicación. Se necesita un poco de humildad pedagógica para hacerlo, pero si no lo hacemos ahora, nuestros alumnos con sus críticas y sarcasmos presentes y futuros, lo harán por nosotros.

\section{Recomendaciones}

Información externa para el diseño del currículo.

Es necesario que tomemos en cuenta directamente a los profesionales en ejercicio cuando se diseña el currículo de una carrera. La fuente de información externa, es la que nos dirá con bastante precisión si las asignaturas diseñadas durante los estudios, han resultado o no pertinentes, en cuanto a su aplicación directa o discretamente directa, cuando el graduado trabaja con su entorno profesional.

Información interna para el diseño del currículo.

En las universidades, generalmente el diseño del currículo es efectuado por un grupo de profesores de la asignatura que está siendo considerada, cuando no lo hace uno solo de los docentes. El sistema de diseño tomando en cuenta solamente la información interna, representa por lo general un estancamiento acomodaticio. En algunas ocasiones la organización de la programación, consiste en la copia directa de un libro, el cual no necesariamente es el más actualizado. Este encerramiento intelectual de algunos docentes se va a reflejar en el futuro, con toda seguridad, en lo que seguimos llamando el currículo nulo, fuente de pérdidas, atrasos y frustraciones. Un diseño curricular que no tome en cuenta a los egresados de la carrera en re programación, está de antemano condenado al fracaso.

\section{REFERENCIAS BIBLIOGRÁFICAS}

1. ARRIETA DE MEZA, Beatriz y MEZA CEPEDA, Rafael. El currículum pertinente del pensum de Idiomas Modernos para el ejercicio profesional del egresado. Laurus Revista de Educación. Vicerrectorado de Docencia. Universidad Pedagógica Experimental Libertador (No. 10). Año 6, p.72-82. Caracas. 2000.

2. CORAZZA, Sandra M. Manual de autoayuda para intelectuales de la educación. Educaçao y Realidade. Currículo, cohecimiento e formaçao docente. Vol. 23 (No. 2), p.43-61. Porto Alegre. Brasil. 1998.

3. EISNER, E. W. The educational imagination Third Edition. New York. MacMillan. U.S.A. 1994.

4. LÓPEZ, N. Retos para la construcción curricular de la certeza al paradigma de la incertidumbre creativa. Cooperativa Editorial Magisterio. Colombia. 1996.

5. MARÍN P, Carlos. Revolución curricular: una experiencia de integración. Universidad Centro Occidental Lisandro Alvarado. Sector Productivo. Ponencia Presentada en la II Reunión Nacional sobre 
Currículo en la Educación Superior. 1995.

6. PEÑALOZA, Walter. El currículo integral. Universidad del Zulia. Vicerrectorado Académico. Maracaibo, Venezuela. 1995.

7. POSNER, George. Análisis de currículum. Segunda Edición. McGraw-Hill Interamericana, S.A. Santa Fe de Bogotá, Colombia. 1998. 